\title{
Atitudes de leitura e desesperança em idosos
}

\author{
Katya Luciane de Oliveira \\ Mirian Cruvinel \\ Universidade Estadual de Campinas, Campinas-SP, Brasil \\ Acácia Aparecida Angeli dos Santos \\ Universidade São Francisco, Itatiba-SP, Brasil
}

\begin{abstract}
Resumo: Nesta pesquisa buscou-se averiguar a relação entre atitudes positivas e negativas em leitura e desesperança em idosos. Participaram deste estudo 79 idosos provenientes de centro de terceira idade, de um posto de retirada de medicamentos e de uma instituição asilar. A média de idade foi de 69 anos e 8 meses. Utilizou-se um questionário para os dados de caracterização dos idosos, o Inventário de Desesperança de Beck e uma escala de atitudes de leitura. Os resultados evidenciaram relação estatisticamente significativa entre a pontuação na escala de atitudes de leitura e o nível de desesperança nos idosos.
\end{abstract}

Palavras-chave: Idoso. Psicologia do envelhecimento. Leitura. Desesperança.

\section{Reading attitude and hopelessness in elderly}

\begin{abstract}
This study investigated the relationship between positive and negative reading attitudes and hopelessness in the elderly. Seventy nine aged individuals, on average 69 years and 8 months old paticipated in the study. They originated from a center for third age people, a medicine distribution facility and from an asylum for the elderly. The material used in the study was a data collecting questionnaire for elderly characterization, the Beck's Despair Scale and a reading attitude scale. The results indicated a significant association between scores in the reading scale and despair level.
\end{abstract}

Keywords: Elderly. Aging psychology. Reading. Hopelessness.

\section{Actitudes en lectura y desesperanza en adultos mayores}

Resumen: En este estudio se buscó investigar la relación entre actitudes positivas y negativas en lectura y desesperanza en ancianos. Participaron del estudio 79 ancianos provenientes del centro de la tercera edad, de un puesto de retirada de medicamentos y de una institución asilar. El promedio de edad fue de 69 años y 8 meses. Se utilizó un cuestionario para los datos de caracterización de los ancianos, el Inventario de Desesperanza de Beck y una escala de actitudes de lectura. Los resultados evidenciaron relación estadísticamente significativa entre la puntuación en la escala de actitudes de lectura y el nivel de desesperanza en los ancianos.

Palabras clave: Anciano. Psicología del envejecimiento. Lectura. Desesperanza. 


\section{Introdução}

Nos primeiros anos do século XX as considerações sobre a população idosa eram atinentes apenas à geriatria, visto ser a área de conhecimento que inicialmente descreveu e compilou dados de forma sistemática sobre essa população. Há que se ressaltar, ainda, os estudos gerontológicos que contribuíram significativamente para o desenvolvimento da área, sobretudo porque aquilataram as investigações produzidas. Atualmente o idoso é estudado a partir de uma visão mais holística, que agrega conceitos da biologia, sociologia e psicologia (Neri, 1997; Hendricks \& Achenbaum, 1999).

A etapa final da vida adulta, segundo Neri (1995), somente começou a ser estudada sistematicamente no final dos anos 1950, sendo que as pesquisas sobre life-span, ou curso da vida, emergiram mais claramente como campo de estudo a partir dos anos 1970. A concepção de curso da vida está representada dentro da psicologia do envelhecimento, na qual a velhice é considerada como uma experiência heterogênea, que apresenta ganhos e perdas, num complexo sistema de interação dinâmica.

No Brasil os estudos sobre a velhice têm crescido expressivamente na psicologia. As últimas duas décadas testemunharam um aumento significativo das pesquisas na área da gerontologia e, mais recentemente, na psicologia do envelhecimento. Apesar disso, Freitas, Maruyama, Ferreira e Motta (2002) admitem que o envelhecimento ainda é algo tão misterioso, quanto o início da vida.

A Organização Mundial de Saúde define que a idade de 60 anos é o início da etapa que se denomina terceira idade. Os resultados de uma pesquisa efetuada por Neri (1991), sobre a concepção do início dessa etapa, evidenciaram que os participantes classificaram o seu início aos 60 anos, enquanto outros classificaram o início acima dos 70 anos e por fim muitos afirmaram que a velhice é um estado de espírito. $\mathrm{O}$ estudo concluiu que ser velho é um estado de espírito, condicionado a fatores de personalidade e experiências anteriores relacionadas à velhice.

Sob essa perspectiva, Freitas e cols. (2002) observam que os fatores envolvidos no envelhecer implicam uma multiplicidade de fatores biológicos, sociais e emocionais que vislumbram a pessoa em sua individualidade. Considerar isoladamente aspectos fisiológicos e psicológicos constitui uma abstração uma vez que são processos interdependentes.

A sociedade no processo de envelhecimento, segundo Fraiman (1995), também desempenha um papel importante ao passo que estabelece as normas de comportamento definindo o significado social de cada idade. Desse modo, considera a idade como sendo uma variável que regula o comportamento social e as relações entre os indivíduos e o seu grupo.

Nessa direção, pode-se aventar que a sociedade considera o idoso alguém dependente o que não representa a realidade dessa população. Um exemplo disso foi a investigação de Ramos, Rosa, Oliveira, Medina e Santos (1993) que estudaram o perfil de 1602 idosos de áreas metropolitanas da região sudeste do Brasil. Os dados mostraram que mais da metade da população estudada (53\%) afirmou que tinha autonomia total na realização de atividades da vida diária o que caracteriza a independência do auxílio de outras pessoas.

Mesmo o idoso apresentando comportamento independente em muitos casos, verifica-se que desprazer em relação à vida é comum nessa faixa etária, visto que as emoções possuem estágios diferentes no adulto. No caso do idoso se houver um bom controle cognitivo, com amplo repertório de experiências e contextos, o estado emocional terá maior oportunidade de elaboração, ao passo que a emoção é o filtro que transforma as operações cognitivas. Uma operação emocional mal sucedida dificulta o processo de regulação o que pode ocasionar algum transtorno emocional (Labouvie-Vief, 1999). Nessa direção, Bahls (1999) chama atenção para o fato de que o aumento das doenças mentais tem sido subestimado pelas pesquisas realizadas. Tais estudos consideram a mortalidade e deixam de avaliar a incapacitação (física, social e emocional) gerada pelas patologias mentais.

Portanto, estudos foram realizados visando buscar maiores informações sobre as variáveis psicológicas relacionadas à população idosa. Dentre eles citam-se as investigações acerca da ansiedade 
(Byrne, 2002; Coes, 1991; Dalgalarrondo, 2000; Manela, Katona \& Livingston, 1996; Souza \& Drucker, 2000) e da depressão (Gazalle, Lima, Tavares \& Hallal, 2004; Piccoloto, Wainer, Benvegnú \& Juruena, 2001; Solomon, 2002; Veras \& Coutinho, 1991).

Cruvinel, Oliveira e Santos (2005) realizaram um estudo exploratório com idosos sobre a satisfação com a vida e os sintomas de ansiedade e depressão. A maior parte dos idosos apresentou uma boa percepção em relação a sua vida. Considerando que os dados revelaram que $80 \%(n=26)$ se consideravam felizes em relação a sua vida e apenas $20 \%(n=4)$ relataram se sentirem infelizes. Embora os idosos tenham relatado que se sentiam felizes, sintomas de ansiedade e depressão em níveis leve e moderado foram detectados.

Sintomas relacionados à ansiedade e à depressão nessa etapa da vida podem ocorrer, conforme aponta Prétat (1994), levando ao aparecimento de emoções mais profundas. Desse modo, o aparecimento de sintomas como desesperança e por vezes distúrbios ansiosos e quadros depressivos, podem ser constatados. É importante destacar que a desesperança pode levar à ansiedade e à depressão, o que possivelmente, em longo prazo, levaria a um comprometimento mais sério da saúde mental (Ramos \& cols., 1993; Coutinho, Gontiès, Araújo \& Sá, 2003).

A desesperança poderia estar relacionada às percepções negativas e a um sentimento de fracasso e derrota em relação a si próprio (Beck, Rush, Shaw \& Emery, 1997). Skinner e Vaughan (1985) destacam que ao envelhecer o idoso passa a apresentar diversos fracassos em tarefas que antes apresentava êxito. Assim sendo, as atividades que apresentam obstáculos e possibilidades de fracassos são abandonadas pelo idoso, o que gera uma sensação autoderrotista e por fim a desesperança.

Contudo, estudos sistemáticos com o objetivo de investigar a desesperança na terceira idade ainda são poucos os realizados no país. Nessa direção, Oliveira, Santos, Cruvinel e Neri (2005) buscaram estabelecer relações entre os sintomas de ansiedade, depressão e desesperança na população idosa. Os dados evidenciaram relação significativa e positiva entre as variáveis e no caso da desesperança o nível encontrado na população pesquisada foi considerado moderado.

Outros aspectos pouco investigados são as atitudes positivas e negativas que o idoso pode apresentar frente à leitura e se isso favorece de algum modo sua saúde mental. Hipotetiza-se que tais atitudes podem interferir no curso de vida do indivíduo, principalmente quando a população é idosa. Para Amorim e Alberto (1996), muitos idosos não vivem cada instante de sua vida com força de vontade e muitas vezes são prejudicados por desprazeres. Nesse sentido, o idoso poderia se beneficiar com uma boa leitura ou como fonte de informação ou de satisfação pessoal.

McDowd e Shaw (2000) observam que a atenção que o processo cognitivo da leitura pode melhorar a capacidade de concentração do idoso, sendo que a atenção pode ser considerada um prérequisito para o comportamento eficiente nessa idade. Outro aspecto importante nesse processo é o estímulo à atenção seletiva, podendo a leitura auxiliar na atenção seletiva do idoso, o que possivelmente resultaria em um melhor desempenho na realização de tarefas que exijam essa habilidade.

A habilidade do idoso de aprender e relembrar os fatos pode estar associada a leitura, conforme apontam Zacks, Hasher e Li (2000). Os resultados da pesquisa que realizaram mostram que o hábito de ler jornais, por exemplo, reduz a possibilidade de esquecimento dos fatos, visto que promove a estimulação dos processos cognitivos. A memória do idoso que lê apresenta menos déficits de atenção e quadros de esquecimentos em relação àqueles que não apresentam esse hábito.

Constata-se, então que a leitura é benéfica à saúde mental do idoso. Staudinger e Pasupathi (2000) indicam que diversos fatores estão implicados no processo de regulação cognitivo e emocional do idoso. Nesse sentido, os idosos que apresentam algum prejuízo cognitivo (esquecimentos, lapsos, entre outros), podem ficar mais propensos a sentimentos autoderrotistas e desesperançosos em relação à vida.

Deeg, Kardaun e Forzard (1996) defendem que existe uma relação entre boa saúde mental e comportamento na terceira idade. As estratégias 
comportamentais que os idosos utilizam podem determinar a diferença entre viver essa etapa da vida com qualidade ou sem ela. Portanto, o investimento na realização de atividades cognitivas, como a leitura, pode melhorar a habilidade de reorganizar e regular as informações.

Filipp (1996) observa que a emoção e a motivação trabalham em conjunto para promover uma mobilização da atividade cognitiva que necessita ser constantemente estimulada. Sob esse aspecto, Schaie (1996) estudou o desenvolvimento intelectual na terceira idade. Os dados apontaram que uma série de fatores pode interferir na habilidade cognitiva do idoso, dentre eles estão os quadros patológicos, as doenças cardiovasculares e o contexto social em que vive o idoso. Aponta, ainda, que a maior parte dos idosos mentalmente saudáveis é aquela que mantém um comportamento intelectual ativo.

A qualidade de vida na velhice está condicionada à satisfação, ao envolvimento e ao senso de realização em sua competência social e cognitiva, o que certamente seria corroborada por meio de atitudes positivas em relação à leitura. As virtudes da velhice e o envelhecer com boa qualidade de vida individual e social dependem do equilíbrio existente entre as limitações e as potencialidades da pessoa (Neri, 1995; 2002).

Cabe ao idoso, portanto, conhecer quais são suas limitações e potencialidades, sendo que a leitura poderá ajudá-lo a encarar com mais esperança essa etapa de sua vida. Portanto, avaliar a relação entre desesperança em relação à vida e as atitudes positivas e negativas direcionadas à leitura em idosos foi o objetivo da presente pesquisa.

\section{Método}

\section{Participantes}

Participaram 79 idosos do sul de Minas Gerais, provenientes de centro de terceira idade $(50,6 \%$; $n=40)$, de um posto de retirada de medicamentos $(38 \% ; n=30)$ e de uma instituição asilar $(11,4 \% ; n=9)$. A média de idade foi de 69 anos e oito meses $(D p=6,7)$, a idade máxima constatada neste estudo foi de 84 anos. $\mathrm{O}$ gênero feminino representou 79,7\% $(n=63)$ da amostra e o masculino $20,3 \%(n=16)$.

\section{Instrumentos}

Foi utilizado um Questionário SócioDemográfico. Trata-se de um questionário que contém questões de caracterização dos participantes e que apresenta questionamentos sobre o comportamento de leitura e a utilização do computador/ internet pelo idoso.

As Escalas Beck (1991) também foram utilizadas. As escalas foram traduzidas e adaptadas para a realidade brasileira, por permissão de The Psychological Corporation, U.S.A., por Cunha (2001). Consistem em quatro escalas que mensuram sintomas de ansiedade, depressão, desesperança e ideação suicida (nesta pesquisa utilizou-se somente a Escala de Desesperança).

A Escala de Desesperança Beck apresenta 20 frases, nas quais o sujeito deve assinalar verdadeiro ou falso para cada afirmação apresentada. A classificação crítica de pessimismo, o que indica a desesperança, varia do nível mínimo de desesperança (0-3), nível leve de desesperança (4-8), nível moderado de desesperança (9-14), nível grave (superior a 14).

Utilizou-se também uma escala de atitudes de leitura. A escala é composta de 30 afirmações relacionadas ao comportamento de ler (Silva \& Naher, 1981). A versão utilizada nos idosos sofreu pequenas adaptações, a fim de atender às características da população idosa. Os idosos liam e respondiam o quanto concordavam com o que foi apresentado na afirmação (concordo plenamente, concordo, discordo, discordo plenamente). A pontuação na escala poderia atingir 120 pontos.

\section{Procedimento}

Os dados foram coletados coletivamente na população que freqüentava o centro de terceira idade e individualmente nos idosos que se dirigiam ao posto de retirada de medicamentos e nos idosos asilares. Em todos os casos foi estabelecido um bom rapport entre os pesquisadores e os participantes, momentos antes da aplicação dos instrumentos. 
A coleta nos três grupos ocorreu somente nos idosos que assinaram o Termo de Consentimento Livre e Esclarecido e foi assegurado aos participantes o caráter confidencial do estudo. Cabe ainda destacar que os cuidados éticos tomados na presente pesquisa foram baseados e estão em consonância com a Resolução 196/96 do Conselho Nacional de Saúde (1996).

\section{Resultados}

Os dados foram organizados em planilhas e submetidos à estatística descritiva e inferencial conforme o objetivo do estudo. No que se refere ao comportamento de ler, foi questionado se os idosos tinham o hábito de comprar e ler jornais. Os dados mostraram que $12,7 \%(n=10)$ sempre tinham esse hábito, $19 \%(n=15)$ às vezes compravam e liam, $10 \%$ $(n=8)$ responderam que raramente faziam isso e $40,5 \%(n=32)$ responderam a alternativa nunca. Salienta-se que $14(17,8 \%)$ idosos não responderam à questão.

Também se questionou o hábito de comprar e ler revistas, $15 \%(n=12)$ afirmaram que sempre costumavam comprar e ler revistas, $16,4 \%(n=13)$ responderam que às vezes faziam isso, $11,9 \%(n=9)$ disseram que raramente tinham esse hábito e $36,6 \%$ $(n=29)$ afirmaram que nunca compravam ou liam revistas. $20,1 \%(n=16)$ foi a porcentagem de pessoas que não responderam a questão.

A freqüência com que compravam e liam livros também foi observada. A alternativa sempre comprava e lia livros foi respondida por 7,6\% $(n=6)$ dos idosos, $12,7 \%(n=10)$ disseram que às vezes tinham esse hábito, a categoria raramente foi respondida por $15,2 \%(n=12)$ dos idosos e a categoria nunca por $40,5 \%(n=32)$. Essa questão não foi respondida por $19(24 \%)$ idosos.

Foi perguntado ao idoso se na residência ele utilizava o computados/internet. Os dados mostraram que 7,6\% $(n=6)$ dos idosos utilizavam à internet, $70,9 \%$ $(n=56)$ afirmaram que não utilizavam, pois não tinham computador em casa e $8,9 \%(n=7)$ responderam que até teriam acesso à internet, contudo, embora tivessem computador em casa, não sabiam mexer. A porcentagem de idosos que não responderam à questão foi de 12,6 $(n=10)$.

A escala de atitudes em leitura foi respondida por todos os idosos. A média de pontos na escala foi de $81,4(D p=22,1)$ a pontuação mínima foi de $7 \mathrm{e}$ a máxima 117. No que se refere à desesperança, a média na Escala de Desesperança foi de 5,2 pontos $(D p=3,9)$, a pontuação mínima foi de 0 e a máxima de 17. Salienta-se que 63 idosos $(79,7 \%)$ responderam à Escala de Desesperança e o restante $(20,3 \% ; n=16)$ não.

Para verificar a relação entre atitudes em leitura e desesperança nos idosos recorreu-se à correlação de Pearson. Os dados indicaram que houve relação estatisticamente significativa entre as variáveis, considerando $r=-0,457 \mathrm{e} p=0,000$. A Figura 1 mostra a dispersão dos participantes.

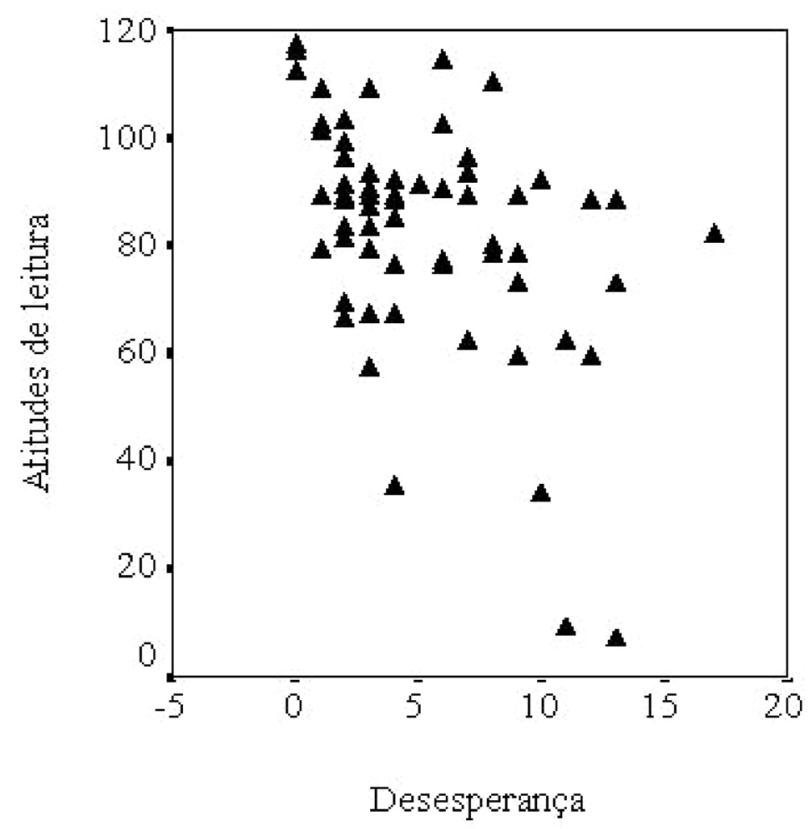

Figura 1. Dispersão dos participantes na relação entre atitudes em leitura e desesperança.

Para verificar se havia diferença na pontuação da escala de atitudes de leitura e os sintomas de desesperança nos idosos recorreu-se à análise de variância. Para tanto, os idosos foram divididos em 
três grupos, idosos com até 73 pontos na escala de atitudes de leitura (G1), idosos com 73 até 91 pontos (G2) e idosos com 92 pontos ou mais na escala (G3).

Os dados mostraram que houve diferença estatisticamente significativa entre os grupos $[F=(2,61)=4,886 ; p=0,01)]$. O teste $a d$-hoc de Tukey revelou que a diferença estava entre os grupos de idosos que apresentaram um resultado de até 73 pontos na escala de atitudes de leitura e aqueles que apresentaram 91 pontos ou mais $(p=0,008)$. A Figura 2 apresenta a distribuição dos participantes por pontuação na escala de atitudes e os sintomas de desesperança.

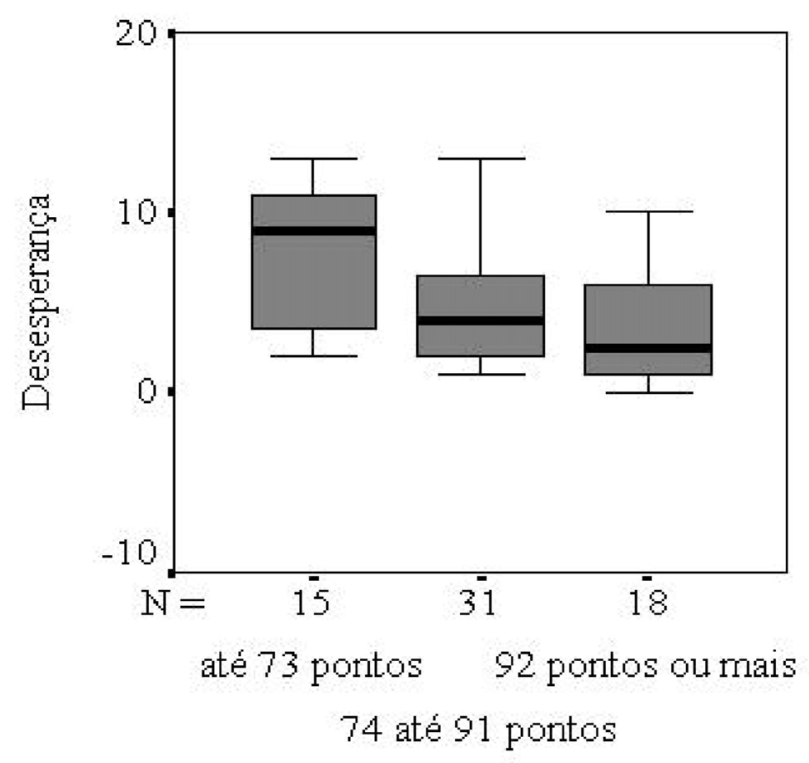

\section{Atitudes de leitura}

Figura 2. Boxplot dos participantes por pontuação na escala de atitudes e os sintomas de desesperança.

Observou-se que não houve diferença significativa entre a desesperança e a pontuação na escala de atitudes em leitura, considerando os idosos do G1 e G3 em relação aos idosos do G2.

\section{Discussão}

A atividade intelectual está relacionada ao comportamento de ler. Na terceira idade essa atividade se beneficia da leitura, ao passo que ao ler o idoso estimula os processos cognitivos o que favorece a diminuição dos lapsos de memória e dos déficits de atenção. Quando os idosos foram questionados sobre a freqüência com que compravam jornais, revistas e livros uma parte deles respondeu que sempre tinha esse hábito. A porcentagem de pessoas que compravam e liam revistas foi maior do que aquelas que compravam e liam jornais ou livros. Contudo, salienta-se que a alternativa sempre não foi assinalada pela maior parte da população o que não é um dado positivo.

Nessa direção, se a leitura pode ser fonte de informação quanto de satisfação pessoal para o idoso (Amorim \& Alberto, 1996), então aqueles que não têm esse hábito deixam de aproveitar de um meio prazeroso como a leitura para extrair do que foi lido reflexões que poderiam gerar novas perspectivas pessoais. Outro aspecto importante de se ler com freqüência nessa etapa da vida é apontado por Zacks e cols. (2000) que defendem que o fato do idoso possuir o hábito diário de ler jornal, seria suficiente para reduzir os quadros de esquecimentos e melhorar a atenção.

Quanto à utilização do computador/internet apenas uma pequena parte da amostra respondeu que utiliza esse recurso. Outra parcela, também reduzida, afirmou que teria acesso à internet na própria casa, mas não sabem como utilizar esse recurso. A maioria dos respondentes afirmou que não tem computador em casa. O computador e a internet poderiam também servir como ferramentas de estímulo cognitivo para o idoso. Entretanto, poucos têm acesso a essa tecnologia para utilizá-la em benefício próprio. Não se pretende encerrar as discussões sobre os efeitos da navegação na rede na saúde mental do idoso, porém sugere-se que o fato de se utilizar mais esse recurso (ligar o computador, acessar a internet, buscar sites e assuntos que interessam) poderia favorecer, de algum modo, a saúde mental do idoso. Sendo assim, como esse não foi o objetivo central deste estudo, sugere-se que outras investigações sejam realizadas, visando elucidar os efeitos da internet no processamento cognitivo do idoso. 
Quanto às atitudes em relação à leitura observou-se que, de uma forma geral, os participantes apresentaram mais atitudes positivas do que negativas em relação à leitura. Esse fato pode ser apoiado na média de pontos apresentada pelos idosos na escala de atitudes em leitura $(M=81,4)$, no entanto o desvio padrão em relação à média foi grande $(D p=22,1)$. Ao que parece quando se considera o escore médio as atitudes positivas prevaleceram, mas ainda se identifica uma dispersão entre a pontuação na escala, permitindo a divisão dos participantes em três grupos. O primeiro grupo foi representado pelos idosos que tiveram até 73 pontos na escala de atitudes de leitura, o segundo por idosos com pontuação de 73 até 91 pontos e o terceiro grupo foi composto por idosos com 92 pontos ou mais na escala.

Percebe-se, então, uma gradação na pontuação da escala, na qual o terceiro grupo seria aquele que apresentou mais atitudes positivas frente à leitura. Embora os idosos tenham apresentado mais atitudes positivas do que negativas em relação à leitura, conforme mostrado nesta pesquisa, esse dado não é sinônimo do comportamento freqüente de ler jornais, revistas ou livros. Aventa-se a hipótese de que faltam mais estímulos aos idosos para realizar a leitura. Seja qual for o motivo apresentar atitudes positivas em relação à leitura pode ser um grande passo para dar início à vida leitora que, segundo McDowd e Shaw (2000), pode melhorar a capacidade de concentração do idoso e funcionar como um pré-requisito para o comportamento eficiente nessa idade.

Quanto à desesperança, a média $(M=5,2)$ apresentada pelos idosos na escala foi correspondente ao nível leve desse sintoma. Esse dado pode ser um indicador de que, de algum modo, a população pesquisada está apresentando sintomas sutis de desesperança. Por óbvio, esse fato pode depor contra a saúde emocional do idoso futuramente, considerando que se nessa etapa da vida as emoções apresentam-se de forma mais latente (Prétat, 1994) e o aparecimento de distúrbios ansiosos e quadros depressivos (Byrne, 2002; Coes, 1991; Cruvinel e cols., 2005; Dalgalarrondo, 2000; Gazalle e cols., 2004; Manela e cols., 1996; Piccoloto e cols., 2001; Ramos e cols., 1993; Solomon, 2002; Souza \& Drucker, 2000;
Veras \& Coutinho, 1991) podem ser diagnosticados. Portanto, os sintomas de desesperança poderiam funcionar como iniciadores de um distúrbio emocional mais severo (Oliveira e cols., 2005).

A relação entre atitudes em leitura e desesperança nos idosos foi evidenciada pela correlação de Pearson. Os idosos que obtiveram uma maior pontuação na escala de atitudes de leitura, o que representa mais atitudes positivas, também foram aqueles que pontuaram menos na escala de desesperança. Em outro sentido, à medida que aumenta as atitudes positivas em relação à leitura diminui o aparecimento de sintomas de desesperança.

A diferença de pontuação na escala de atitudes de leitura e os sintomas de desesperança também foi apontada por meio do teste ad-hoc de Tukey. Novamente o grupo de idosos que obteve uma maior pontuação na escala, também foi o grupo que apresentou menos sintomas desesperançosos em relação aos que pontuaram menos.

Esses dados corroboram o fato de que o processo cognitivo da leitura promove a estimulação da concentração (Zacks e cols., 2000), da atenção seletiva e do relembrar, sendo que os lapsos e os déficits de memória tornam-se menos freqüentes (McDowd \& Shaw, 2000). Outros aspectos importantes relacionados ao comportamento de ler são a regulação e a organização de dados (Deeg e cols., 1996), a emoção e a motivação (Filipp, 1996) e o aprimoramento das habilidades intelectuais (Schaie, 1996).

No que se refere à esperança direcionada ao viver, Skinner e Vaughan (1985) entendem que o fracasso nessa etapa da vida pode gerar no idoso sentimentos negativos em relação à vida e a si próprio. Em acréscimo, Staudinger e Pasupathi (2000) destacam que a leitura pode beneficiar a saúde mental e emocional do idoso por estimular a cognição. Nesse contexto, com a melhora da competência cognitiva, os aspectos emocionais também sofreriam um equilíbrio, os idosos ficariam mais esperançosos em relação a sua vida e a qualidade de vida poderia aumentar (Neri, 2002). 


\section{Considerações finais}

Os déficits cognitivos diminuem nos idosos que têm o hábito de ler, sendo que esses déficits podem incitar nos idosos o aparecimento de sentimentos negativos e de baixa auto-estima o que inevitavelmente poderia levar a quadros como a desesperança e mais tarde à ansiedade e à depressão. Assim, conhecendo a importância da leitura na terceira idade, os centros e os serviços especializados em atender essa população deveriam investir mais recursos com o intuito de incentivar a leitura nessa etapa da vida.

Talvez o idoso tenha vontade de ler, mas diversos fatores podem impedir a ocorrência desse comportamento. Dentre eles pode-se citar a falta de recursos financeiros para comprar o material, a carência de orientações para freqüentar bibliotecas, ou ainda a ausência de publicações especializadas (revistas, jornais e livros) que interessariam a essa população.

Algumas iniciativas como serviços diferenciados em bancos (prêmios para talentos da terceira idade, contas especiais, empréstimos, atendimentos preferenciais), linhas de produtos de beleza destinados à terceira idade, dentre outras, estão sendo implementadas. Mas, essas iniciativas ainda são poucas e, de uma forma geral, são fomentadas por empresas privadas. Atualmente, se desconhece a existência de programas de abrangência nacional, sejam públicos ou privados que invistam em leitura para os idosos. Outros aspectos poderiam ser mencionados nesta discussão, todavia escapam do foco da presente pesquisa.

Desse modo, a realização de investigações sistemáticas sobre o assunto, poderia subsidiar novas linhas de análise desse fato. Finalmente, cabe destacar que novos estudos deverão ser realizados, a fim de explorar melhor a desesperança e a leitura na terceira idade, bem como buscar diversificar a amostra e ampliar a coleta de dados para outras regiões do país.

\section{Referências}

Amorim, C. A., \& Alberto, C. A. (1996). Atividades físicas e terceira idade: uma perspectiva psicossocial. Psicologia Argumento, 14, 47-64.
Bahls, S. C. (1999). Depressão: uma breve revisão dos fundamentos biológicos e cognitivos. InterAção, 3, 49-60.

Beck. A T., Rush A J., Shaw, B. F., \& Emery, G. (1997). Terapia cognitiva da depressão (Sandra Costa, trad.). Porto Alegre: Artes Médicas.

Byrne, G. (2002). What happens to anxiety disorders in later life? Revista Brasileira de Psiquiatria, 24(Supl. 1), 74-80.

Coes, M. C. R. (1991). Ansiedade: uma avaliação quantitativa de seus efeitos negativos sobre o desempenho no vestibular. Psicologia: Teoria e Pesquisa, 7, 137-147.

Conselho Nacional de Saúde. (1996, 10 de outubro). Resolução 196/96. Diretrizes e normas regulamentadoras de pesquisa. Retirado em 19 de outubro de 2005, de http:// www.conselho.saude.gov.br/resolucoes/1996/ Reso196.doc

Coutinho, M. P. L., Gontiès, B., Araújo, L. F. \& Sá, R. C. N. (2003). Depressão, um sofrimento sem fronteira: representações sociais entre crianças e idosos, Psico-USF, 8(2), 183-192.

Cunha, J. (2001). Manual da versão em português das escalas Beck. São Paulo: Casa do Psicólogo.

Cruvinel, M., Oliveira, K. L., \& Santos, A. A. A. (2005). Avaliação psicológica em idosos: Um estudo sobre hábitos saudáveis, ansiedade e depressão [CD-ROM]. In II Congresso Brasileiro de Avaliação Psicológica: Desafios para a formação, prática e pesquisa. Gramado: Instituto Brasileiro de Avaliação Psicológica.

Dalgalarrondo, P. (2000). Psicopatologia e semiologia dos transtornos mentais. Porto Alegre: Artmed.

Deeg, D. J. H., Kardaun, J. W. P. F., \& Forzard, J. L. (1996). Health, behavior and aging. In J. E. Birren, K. W. Schaie, R. P. Abeles, M. Gatz \& T. J. Salthouse (Eds.), Handbook of psychology of aging ( $4^{\text {th }}$ ed., pp. 129-149). London: Academic Press. 
Filipp, S. H. (1996). Motivation and emotion. In J. E. Birren, K. W. Schaie, R. P. Abeles, M. Gatz \& T. J. Salthouse (Eds.), Handbook of psychology of aging ( $4^{\text {th }}$ ed., pp. 219-235). London: Academic Press.

Fraiman, A. P. (1995). Coisas da idade (3 ${ }^{\mathrm{a}}$ ed.). São Paulo: Gente.

Freitas, M. C., Maruyama, S. A. T., Ferreira, F. T., \& Motta, A. M. A. (2002). Perspectivas das pesquisas em gerontologia e geriatria: revisão da literatura. Revista Latino-Americana de Enfermagem, 10, 221-228.

Gazalle, F. K., Lima, M. S, Tavares, B. F., \& Hallal, P. C. (2004). Sintomas depressivos e fatores associados em população idosa no sul do Brasil. Revista de Saúde Pública, 38, 365-71.

Hendricks, J., \& Achenbaum, A. (1999). Historical development of theories of aging. In V. L. Bengtson \& K. W. Schaie (Eds.), Handbook of theories of aging (pp. 21-39). New York: Springer.

Labouvie-Vief, G. (1999). Emotions in adulthood. In V. L. Bengtson \& K. W. Schaie (Eds.), Handbook of theories of aging (pp. 253-267). New York: Springer.

Manela, M., Katona, C., \& Livingston, G. (1996). How common are the anxiety disorders in old age? Geriatric Psychiatry, 11(1), 65-70.

McDowd, J. M., \& Shaw, R. (2000). Attention and aging: a functional perspective. In F. I. M. Craik \& T. A. Salthouse (Eds.), The handbook of aging and cognition (pp. 221-292). Mahway: Lawrence Erlbaum Associates.

Neri, A. L. (1991). Envelhecer num país de jovens. Significados de velho e velhice segundo brasileiros não idosos. Campinas, SP: UNICAMP.

Neri, A. L. (1995). Psicologia do envelhecimento: uma área emergente. In A. L. Néri (Org.), Psicologia do envelhecimento: Temas selecionados na perspectiva de curso de vida (pp.13-40). Campinas, SP: Papirus.
Neri, A. L. (1997). A pesquisa em gerontologia no Brasil: análise de conteúdos de amostra de pesquisa no período de 1975-1996. Texto e Contexto, 6, 69-105.

Neri, A. L. (2002). Qualidade de vida na velhice. In M. Delitti (Org.), Sobre comportamento e cognição: Vol. 2. A prática da análise do comportamento e da terapia cognitivocomportamental (pp. 282-285). Santo André, SP: ESETec.

Oliveira, K. L., Santos, A. A. A., Cruvinel, M., \& Neri, A. L. (2005). Relação entre ansiedade, depressão e desesperança entre grupos de idosos. Psicologia em Estudo, 11, 351-359.

Piccoloto, N. Wainer, R., Benvegnú, L. \& Juruena, M. (2001). Curso e prognóstico da depressão. Revisão comparativa entre os transtornos do humor [versão eletrônica]. Revista de Psiquiatria Clínica, 27(2). Retirado em 21 setembro 2004, de http://www.honet.usp.br/ipq/revista/r27(2)/ art93.htm

Prétat, J. R. (1994). Coming to age: The croning years and late-life transformation. Toronto: Inner City Books.

Ramos, L. R., Rosa, T. E. C., Oliveira, Z. M., Medina, M. C. G., \& Santos, F. R. G. (1993). Perfil do idoso em área metropolitana na região sudeste do Brasil: resultados de inquérito domiciliar. Revista de Saúde Pública, 27, 87-94.

Schaie, K. W. (1996). Intellectual development in adulthood. In J. E. Birren, K. W. Schaie, R. P. Abeles, M. Gatz \& T. J. Salthouse (Eds.), Handbook of psychology of aging ( $4^{\text {th }}$ ed., pp. 266-286). London: Academic Press.

Silva, E. T., \& Naher, J. P. (1981). Questionário para avaliar atitudes de leitura de alunos de $1^{\circ}$ e $2^{\circ}$ graus (pp. 19-26). Livro de Resumos do III Congresso de Leitura do Brasil. Campinas, SP: UNICAMP.

Skinner, B. F., \& Vaughan, M. E. (1985). Viva bem a velhice: Aprendendo a programar a sua vida (A. L. Neri, trad.). São Paulo: Summus. 
Solomon, A. (2002). O Demônio do meio-dia: Uma anatomia da depressão. Rio de Janeiro: Objetiva.

Souza, A. M., \& Drucker, C. (2000). Terapia cognitivo-comportamental nos transtornos ansiosos no idoso (56-57). Livro de Resumos do IX Encontro Brasileiro de Psicoterapia e Medicina Comportamental. Campinas, SP.

Staudinger, U. M., \& Pasupathi, M. (2000). Life-span perspectives on self, personality and social cognition. In F. I. M. Craik \& T. A. Salthouse (Eds.), The handbook of aging and cognition (pp. 633-688). Mahway: Lawrence Erlbaum Associates.

Veras, R. P., \& Coutinho, E. S. F. (1991). Estudo de prevalência de depressão e síndrome cerebral orgânica na população de idosos. Revista de Saúde Pública, 25, 209-217.

Zacks, R. T., Hasher, L., \& Li, K. Z. H. (2000). Human memory. In F. I. M. Craik \& T. A. Salthouse (Eds.), The handbook of aging and cognition (pp. 293-357). Mahway: Lawrence Erlbaum Associates.

Artigo recebido em 22/05/2006.

Aceito para publicação em 24/08/2007.

Endereço para correspondência:

Katya Luciane de Oliveira. Universidade São Francisco. Rua Alexandre Rodrigues Barbosa, 45, Centro, CEP: 13251-900. Itatiba-SP, Brasil. E-mail: katya.oliveira@saofrancisco.edu.br

Katya Luciane de Oliveira é Psicóloga; Mestre em Psicologia pelo Programa de Pósgraduação Stricto-sensu em Psicologia da Universidade São Francisco; doutoranda em Psicologia, Desenvolvimento Humano e Educação pela Faculdade de Educação da Universidade Estadual de Campinas. Docente do curso de Psicologia da Universidade São Francisco.
Mirian Cruvinel é Psicóloga; Mestre em Educação e doutoranda em Psicologia, Desenvolvimento Humano e Educação, ambos pela Faculdade de Educação da Universidade Estadual de Campinas.

Acácia Aparecida Angeli dos Santos é Psicóloga; Doutora em Psicologia Escolar e do Desenvolvimento Humano pela Universidade de São Paulo. Docente da graduação no curso de Psicologia e no Programa de Pós-Graduação Stricto-sensu em Psicologia da Universidade São Francisco. Bolsista de Produtividade em Pesquisa do CNPq. 